\title{
The morphological progression of viral myocarditis
}

\author{
M.E. Billingham and H.D. Tazelaar \\ Stanford University Medical Center, Stanford, California 94305, USA.
}

\begin{abstract}
Summary: In an attempt to document the morphological progression from acute idiopathic myocarditis to end-stage dilated cardiomyopathy we studied 20 patients with a diagnosis of myocarditis who had had serial endomyocardial biopsies performed with intervals of 1 month to 2 years and whose ages varied from 6 months to 62 years. Fifteen of these patients were treated with immunosuppressive drugs for myocarditis. Ten out of 15 treated patients stabilized clinically. In the remaining 5 cases there was worsening congestive heart failure and 1 patient underwent cardiac transplantation. Of the 5 patients who did not receive immunosuppression, 2 stabilized spontaneously, and 3 developed heart failure, 2 of whom subsequently had cardiac transplants. Whether the patients received immunosuppression or not, in all cases, the inflammatory infiltrate was less but the myocardium developed significant hypertrophy with an increase in interstitial fibrosis and in 8 cases the morphological changes were those of dilated cardiomyopathy.

From the morphological standpoint of this study we have shown some evidence that dilated cardiomyopathy can be the end result of acute myocarditis. It appears that not every case of acute myocarditis progresses to dilated cardiomyopathy and that steroid treatment does not necessarily prevent progression of myocarditis to dilated cardiomyopathy.
\end{abstract}

\section{Introduction}

It has long been suspected that viral myocarditis may lead to idiopathic dilated cardiomyopathy. There is now a growing body of evidence associating serological findings consistent with Coxsackie B viral subtypes with the development of acute myocarditis and a smaller number associating it with idiopathic dilated cardiomyopathy (Kereiakes \& Parmley, 1984). Recent reports have shown that morphological demonstration of acute myocarditis is much lower than that suspected clinically (Billingham \& Mason, 1984) so despite the availability of adequate follow-up series in active myocarditis, there is a paucity of reports documenting morphological progression from viral myocarditis to idiopathic dilated cardiomyopathy (O'Connell, 1983). There have also been reports that immunosuppressive agents may improve or stabilize the clinical course of myocarditis and that they might prevent the development of idiopathic dilated cardiomyopathy (Mason \& Billingham, 1980). Since the advent of endomyocardial biopsy it has been possible to study the course of myocarditis so that progression to idiopathic dilated cardiomyopathy or changes induced by immunosuppression can be documented morphologically by serial endomyocardial biopsies. The purpose of this study is to examine the subsequent

Correspondence: M.E. Billingham, M.B.B.S., M.R.C.Path, F.C.A.P., F.A.C.C. serial biopsies in patients from our medical centre in whom the first biopsy showed evidence of an active myocarditis, to try to document the morphological progression to idiopathic dilated cardiomyopathy. Looking at the other end of the spectrum, we have also examined the hearts of end-stage cases of idiopathic dilated cardiomyopathy in an attempt to document that active myocarditis had been present previously (Tazelaar \& Billingham, 1985).

\section{Patients and methods}

Twenty patients, 7 males and 13 females with an age range of 6 months to 62 years (mean 33.7) were studied. These were the only patients in our records who had had two or more serial endomyocardial biopsies, the first of which had a diagnosis of acute myocarditis. The number of serial biopsies in this group varied from 2-7 per patient (mean 2.8). The time range between the first and last biopsy varied from one month to 12 months (mean 5 months). Fifteen of the 20 study patients were treated with azathioprine and prednisone for 4-6 weeks following the first biopsy. The usual treatment consisted of prednisone $1.25 \mathrm{mg} / \mathrm{kg} /$ day and azathioprine $2.0 \mathrm{mg} /$ $\mathrm{kg} /$ day for one week then dose tapering for 3-6 weeks.

All the biopsies were stained with haematoxylin and 
eosin as well as with Masson's trichrome to highlight myocyte necrosis and fibrosis. All these patients were biopsied originally for cardiomegaly and congestive heart failure. The endomyocardial biopsies were performed by the previously described method (Caves $e t$ al., 1973) of percutaneous, transjugular right ventricular biopsy using the Stanford-Caves-Schultz bioptome. The initial diagnosis of myocarditis required a definite interstitial inflammatory infiltrate with myocyte damage and essentially normal myocardial morphology (Figure 1). The second and subsequent biopsies were scored on a scale of 1-3 for myocyte hypertrophy and increased interstitial fibrosis. A score of 1 denoted normal myocardial width with normal sized bland, oval nuclei and no increase in interstitial fibrosis. A score of 2 denoted definite myocyte hypertrophy with larger, hyperchromatic nuclei and a moderate increase in interstitial fibrosis. A score of 3 was given when there was marked myocyte hypertrophy with bizarre-shaped hyperchromatic nuclei, mixed with attenuated myocytes (loss of myofibrils confirmed by electron microscopy) and a marked increase in interstitial fibrosis (consistent with changes seen in idiopathic dilated cardiomyopathy). Morphometric estimation of these features was not made.

We also examined the hearts of 108 cases of endstage idiopathic dilated cardiomyopathy for evidence of previous myocarditis (Tazelaar \& Billingham, 1985). These hearts were the recipient (native) hearts of 108 cardiac allograft recipients aged $12-55$ years (mean 33.5 years). All these patients had grossly enlarged globular hearts without evidence of coronary artery or valvular disease. All were in New York Heart Association Class 4 clinically before transplantation. A mean of 6 large random sections (minimum 3) were studied

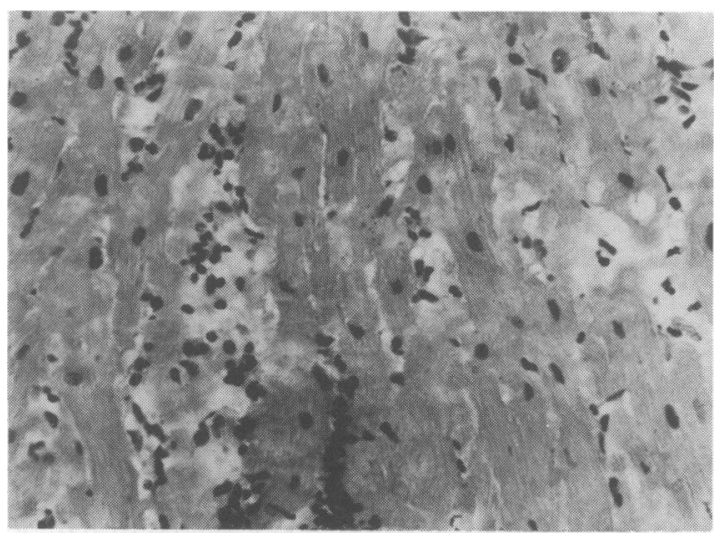

Figure 1 Endomyocardial biopsy showing a lymphocytic infiltration and myocyte damage consistent with myocarditis. Haematoxylin and eosin $\times 320$. from each heart to document the presence of inflammatory infiltrates and their location within the heart. The infiltrates were graded by the numbers of foci seen. Each focus consisted of a minimum cluster of 5 lymphocytes; however, in most cases they were of much larger numbers of cells. The sections were also studied for evidence of a previous pericarditis.

\section{Results}

The results of this study are shown in part in Table I. Of the 15 patients who received immunosuppressive treatment following the diagnosis of myocarditis, 10 stabilized clinically but in each case there was a progression of the score for myocyte hypertrophy and fibrosis in the subsequent biopsies. In all cases the lymphocytic infiltrate was reduced. Of the five patients who developed a morphological score of 3 , consistent with idiopathic dilated cardiomyopathy (Figure 2), two developed worsening congestive heart failure, one received a cardiac transplant and two died.

Of the five patients who did not receive immunosuppressive drugs, two 'resolved' spontaneously, three developed worsening congestive heart failure and subsequently two had cardiac transplants for idiopathic dilated cardiomyopathy.

The results of the study of 108 hearts of end-stage idiopathic dilated cardiomyopathy for the presence of previous myocarditis are shown in Table II. It can bec seen that $87 \%$ showed inflammatory infiltrates predominantly lymphocytic. Only $9.3 \%$ showed residual lymphocytes in the pericardium but in none was there good evidence of a previous pericarditis. As shown in Table II, $32 \%$ of the hearts showed $1-5$ foci of inflammatory cells, $47 \%$ showed $6-30$ foci, and

Table I Results of the morphological progression from myocarditis

\begin{tabular}{lcc}
\hline & & $\begin{array}{c}\text { Score* of progression } \\
\text { by endomyocardial } \\
\text { biopsy }\end{array}$ \\
\hline Yes & No. patients & $1 \rightarrow 2$ \\
Yes & 10 & $1 \rightarrow 3$ \\
Yes & 3 & $2 \rightarrow 3$ \\
No & 2 & $1 \rightarrow 2$ \\
No & 2 & $1 \rightarrow 3$ \\
No & 1 & $2 \rightarrow 3$ \\
\hline
\end{tabular}

*Score 1 = No myocyte hypertrophy and no increase in fibrosis; 2 = moderate myocyte hypertrophy and moderate increase in fibrosis; $3=$ severe myocyte hypertrophy and severe increase in fibrosis. 


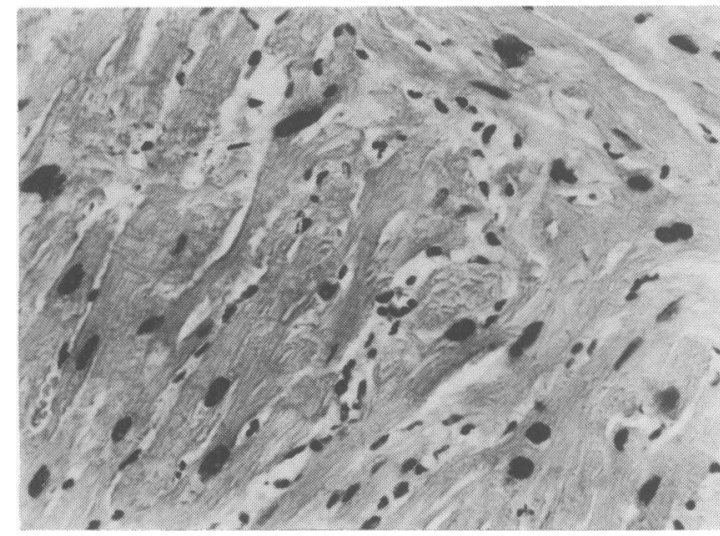

Figure 2 Endomyocardial biopsy from the same patient as in Figure 1, showing progression to myocyte hypertrophy, fibrosis and changes consistent with idiopathic dilated cardiomyopathy. Haematoxylin and eosin $\times 320$.

$7.5 \%$ showed greater than 30 foci of inflammatory cells. The distribution of these infiltrates is described in greater detail by Tazelaar \& Billingham (1986).

\section{Discussion}

Although the numbers are too small to draw conclusions it appears that in some cases myocarditis can resolve spontaneously and that in other cases it can progress to idiopathic dilated cardiomyopathy whether or not immunosuppression is given. Although immunosuppression reduces the inflammatory infiltrate it does not prevent the development of fibrosis or myocyte hypertrophy and attenuation leading to ventricular dilatation and congestive heart failure. The use of immunosuppression to treat active myocarditis is still controversial and evidence that it is helpful in this condition is still inconclusive and is based on a few case reports, as in this study. Until a prospective randomized trial is performed this question is likely to remain unresolved.

There are also anecdotal reports of myocarditis progressing to end-stage idiopathic dilated cardiomyopathy (O'Connell, 1983) and this study adds
Table II Inflammatory infiltrates found in the hearts of 108 patients with idiopathic dilated cardiomyopathy (IDCM)

$\begin{array}{lrl}\text { Total } & 84 & (87 \%) \\ \text { 1-5 foci } & 35 & (32 \%) \\ \text { 6-30 foci } & 51 & (47 \%) \\ \text { >30 foci } & 8 & (7.5 \%) \\ \text { Mixed } & 17 & (16 \%) \\ \text { Pericardial } & 10 & (9.3 \%)\end{array}$

evidence of a few more. We also approached the problem from the end result of idiopathic dilated cardiomyopathy to assess whether or not there was residual morphological evidence of a previous myocarditis. Our group of explanted hearts had the advantage over an autopsy study in that the hearts were examined completely fresh and unlike most autopsy cases, the hearts were from patients free of bronchopneumonia or sepsis since this would have precluded them as heart transplant recipients. We were surprised therefore to find that $87 \%$ of the hearts contained lymphocytic aggregates suggesting a possible previous myocarditis. Against this theory, however, is the fact that none of the 108 cases studied showed good evidence of a healed pericarditis although $9.3 \%$ did have residual pericardial lymphocytic infiltrates. Although cases of florid hypersensitivity myocarditis has been documented by cardiac biopsy none of these have been shown to progress to endstage idiopathic dilated cardiomyopathy even though the degree of inflammatory cells and myocyte necrosis is often more severe. In the same way, acute cardiac rejection, which is a florid lymphocytic myocarditis, has not resulted in a dilated cardiomyopathy in any of the hundreds of cardiac transplant survivors, some of whom are 14 and 15 years post-transplantation.

In conclusion, although the number of patients is small, this study does indicate that there is a subset of patients with acute myocarditis who have progression to idiopathic dilated cardiomyopathy even if treated with immunosuppression. On the other hand there is as yet no conclusive evidence that every case of idiopathic dilated cardiomyopathy results from an episode of acute myocarditis or that every case of acute myocarditis progresses to idiopathic dilated cardiomyopathy.

\section{References}

BILLINGHAM, M.E. \& MASON, J.W. (1984). Endomyocardial biopsy diagnosis of myocarditis. In Viral Heart Disease, H.D. Bolte (ed). p. 201. Springer-Verlag: Berlin.

CAVES, P.K., STINSON, E.B., BILLINGHAM, M.E. \& SHUM-
WAY, N.E. (1973). Percutaneous transvenous endomyocardial biopsy in human heart recipients (experience with a new technique). Annals of Thoracic Surgery, 16, 325. KEREIAKES, D.J. \& PARMLEY, W.W. (1984). Myocarditis 
and cardiomyopathy. American Heart Journal, 108, 1318. MASON, J.W. \& BILLINGHAM, M.E. (1980). Treatment of acute myocarditis assisted by endomyocardial biopsy. American Journal of Cardiology, 45, 1037.

O'CONNELL, J.B. (1983). Evidence linking viral myocarditis to dilated cardiomyopathy in humans. In Myocarditis:
Precursor of Cardiomyopathy, Robinson, J.A. \& O'Connell, J.B. (eds). p. 96. The Collamore Press.

TAZELAAR, H.D. \& BILLINGHAM, M.E. (1986). Leucocytic infiltrates in idiopathic dilated cardiomyopathy: A source of confusion with active myocarditis American Journal of Surgical Pathology (in press). 